\title{
Axisymmetric bending of a circular three-layer plate taking into account creep
}

\author{
Anton Chepurnenko ${ }^{1 *}$, Dmitriy $\mathrm{V}$ ysokovskiy ${ }^{1}$, Elizaveta $R$ usakova ${ }^{1}$, Vladimir $\mathrm{V}$ anja ${ }^{2}$, and \\ Serdar Y azyev $^{1}$ \\ ${ }^{1}$ D on State Technical University, 344002, Rostov-on-D on, Russia \\ ${ }^{2} \mathrm{~K}$ uban State A grarian University named after I.T. Trubulin, 350044, K rasnodar, R ussia
}

\begin{abstract}
The article discusses a method for calculating three-layer plates, in which a lightweight viscoelastic material acts as a middle layer. The technical theory of three-layer structures is used. The derivation of the resolving equation, the numerical and analytical solution of the problem, as well as comparison with the solution in the LIRA software package are presented.
\end{abstract}

\section{Introduction}

Three-layer plates and shells, combining lightness and high load-bearing capacity, are now widely used in construction. It is known that polyurethane foam, which is used in such structures as a middle layer, has not only elastic properties, but also viscosity. Therefore, for an adequate description of the stress-strain state of three-layer structures, it is necessary to use the apparatus of the creep theory. Most of the existing publications on the creep of three-layer structures refer to beams [1-4]. The purpose of this work is to develop the method for calculating circular axisymmetrically loaded three-layer plates with a lightweight filler, taking into account creep.

\section{Methods}

The element of the plate under consideration is shown in Figure 1. In the calculation, we will assume that the viscoelastic properties are characteristic only of the middle layer, and the sheathings work elastically.

Sheathings deformations are defined as follows:

$$
\begin{gathered}
\varepsilon_{r}^{+(-)}=\frac{\partial u^{+(-)}}{\partial r} \\
\varepsilon_{\theta}^{+(-)}=\frac{u}{r}
\end{gathered}
$$

\footnotetext{
${ }^{*}$ Corresponding author: anton_chepurnenk@ mail.ru
} 
Here, the plus corresponds to the bottom sheathing, and the minus to the top.

$$
\begin{aligned}
& \sigma_{r}^{+(-)}=\frac{E}{1-v^{2}}\left(\varepsilon_{r}^{+(-)}+v \varepsilon_{\theta}^{+(-)}\right) ; \\
& \sigma_{\theta}^{+(-)}=\frac{E}{1-v^{2}}\left(\varepsilon_{\theta}^{+(-)}+v \varepsilon_{r}^{+(-)}\right) .
\end{aligned}
$$

The bending moments are calculated as follows:

$$
\begin{aligned}
& M_{r}=\left(\sigma_{r}^{+}-\sigma_{r}^{-}\right) \delta \frac{h}{2} \\
& M_{\theta}=\left(\sigma_{\theta}^{+}-\sigma_{\theta}^{-}\right) \delta \frac{h}{2} .
\end{aligned}
$$

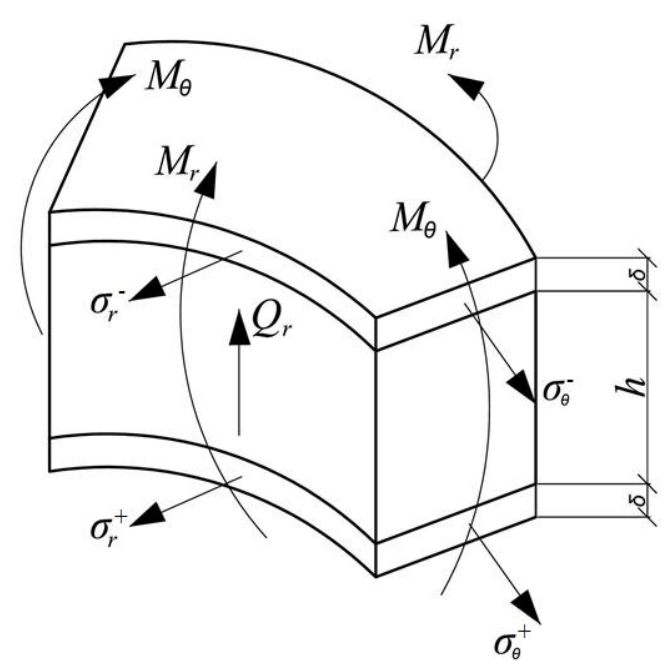

Fig. 1. Three-layer circular plate el ement

Substituting (1) in (2) and then (2) in (3), we get:

$$
\begin{aligned}
& M_{r}=\frac{E \delta h}{2\left(1-v^{2}\right)}\left(\frac{\partial}{\partial r}\left(u^{+}-u^{-}\right)+\frac{v}{r}\left(u^{+}-u^{-}\right)\right) \\
& M_{\theta}=\frac{E \delta h}{2\left(1-v^{2}\right)}\left(v \frac{\partial}{\partial r}\left(u^{+}-u^{-}\right)+\frac{u^{+}-u^{-}}{r}\right)
\end{aligned}
$$

Introducing the value $\alpha=\left(u^{+}-u^{-}\right) / h$, relations (4) can be rewritten as: 


$$
\begin{aligned}
& M_{r}=D\left(\frac{\partial \alpha}{\partial r}+v \frac{\alpha}{r}\right) \\
& M_{\theta}=D\left(v \frac{\partial \alpha}{\partial r}+\frac{\alpha}{r}\right)
\end{aligned}
$$

where $D=\frac{E \delta h^{2}}{2\left(1-v^{2}\right)}$ - cylindrical stiffness of the three-layer plate.

For the displacements of the filler, we take a linear distribution in thickness:

$$
u_{m}=\frac{u^{+}+u^{-}}{2}+\frac{u^{+}-u^{-}}{h} z=u+\alpha z
$$

where $u$ - displacements in the median plane.

Total shear strains in the filler are calculated as follows:

$$
\gamma_{r z}^{m}=\frac{\partial u^{m}}{\partial z}+\frac{\partial w}{\partial r}=\alpha+\frac{\partial w}{\partial r}
$$

On the other hand, the total strain is the sum of the elastic strain and the creep strain:

$$
\gamma_{r z}^{m}=\frac{\tau_{r z}}{G_{m}}+\gamma_{r z}^{*}
$$

where $G_{m}$ - filler shear modulus

L et us express the shear stresses from (8):

$$
\tau_{r z}=G_{3}\left(\gamma_{r z}^{m}-\gamma_{r z}^{*}\right)=G_{m}\left(\alpha+\frac{\partial w}{\partial r}-\gamma_{r z}^{*}\right)
$$

Taking into account the hypothesis of a uniform distribution of shear stresses over the thickness of the aggregate, the shear force $Q$ is written in the form:

$$
Q_{r}=\tau_{r z} h=G_{m} h\left(\alpha+\frac{\partial w}{\partial r}-\gamma_{r z}^{*}\right)
$$

Let us express the value of $\alpha$ from (10) and substitute it into (5):

$$
\begin{aligned}
& M_{r}=D\left(-\frac{\partial^{2} w}{\partial r^{2}}-\frac{v}{r} \frac{\partial w}{\partial r}+\frac{\partial \gamma_{r z}^{*}}{\partial r}+\frac{v}{r} \gamma_{r z}^{*}+\frac{1}{G_{3} h}\left(\frac{\partial Q}{\partial r}+v \frac{Q}{r}\right)\right) \\
& M_{\theta}=D\left(-v \frac{\partial^{2} w}{\partial r^{2}}-\frac{1}{r} \frac{\partial w}{\partial r}+v \frac{\partial \gamma_{r z}^{*}}{\partial r}+\frac{\gamma_{r z}^{*}}{r}+\frac{1}{G_{3} h}\left(v \frac{\partial Q}{\partial r}+\frac{Q}{r}\right)\right) .
\end{aligned}
$$


Equilibrium equations in the case of axisymmetric bending have the form:

$$
\begin{gathered}
\frac{\partial}{\partial r}(Q r)=-q r ; \\
\frac{\partial M_{r}}{\partial r}+\frac{M_{r}}{r}-\frac{M_{\theta}}{r}=Q .
\end{gathered}
$$

The shear force can be determined from the first equation in (12):

$$
Q=-\frac{1}{r} \int_{0}^{r} q(r) r d r
$$

It can be seen from equation (13) that the shear force, and hence the shear stresses in the filler, do not change during the creep process.

To obtain the resolving equation, we substitute the expressions for bending moments (11) into the second equilibrium equation (12). As a result, we get the following equation:

$$
\frac{\partial^{3} w}{\partial r^{3}}+\frac{1}{r} \frac{\partial^{2} w}{\partial r^{2}}-\frac{1}{r^{2}} \frac{\partial w}{\partial r}=-\frac{Q}{D}-\frac{1}{G_{m} h} \frac{\partial q}{\partial r}+\frac{\partial}{\partial r}\left(\frac{1}{r} \frac{\partial}{\partial r}\left(r \gamma_{r z}^{*}\right)\right) .
$$

The elastic problem at $q=$ const can be solved analytically. Equation (14) in this case can be written in the form:

$$
\frac{d}{d r}\left[\frac{1}{r} \frac{d}{d r}(\varphi r)\right]=-\frac{Q}{D}
$$

$\varphi=\frac{d w}{d r}$ - angle of rotation of the normal.
where
The shear force at $q=$ const for a plate without a hole is

$$
Q=-\frac{q r}{2} .
$$

Integrating equation (16), we get:

$$
w=\frac{q r^{4}}{64 D}+\frac{\tilde{C}_{1} r^{2}}{4}+C_{2} \ln r+C_{3}=\frac{q r^{4}}{64 D}+C_{1} r^{2}+C_{2} \ln r+C_{3} .
$$

For a plate without a hole, the constant $C_{2}$ must be zero, otherwise at $r=0$ the deflection will be undefined. Constants $C_{1}$ and $C_{3}$ are determined from the boundary conditions on the contour at $r=R$.

For a hinge-supported along the contour plate: 


$$
\begin{gathered}
w(R)=0 \rightarrow \frac{q R^{4}}{64 D}+C_{1} R^{2}+C_{3}=0 \\
M_{r}(R)=0 \rightarrow \frac{3 q R^{2}}{16 D}+2 C_{1}+v \frac{q R^{2}}{16 D}+2 C_{1} v=-\frac{q(1+v)}{2 G_{m} h} .
\end{gathered}
$$

A fter transformations from (18) we get:

$$
C_{1}=-\frac{q}{4 G_{m} h}-\frac{(3+v)}{(1+v)} \frac{q R^{2}}{32 D} ; C_{3}=\frac{q R^{2}}{4 G_{m} h}+\frac{q R^{4}}{64 D} \frac{(5+v)}{(1+v)}
$$

The deflection at the center of the plate can be calculated using the formula:

$$
w_{\max }=w(0)=C_{3}=\frac{q R^{4}}{64 D} \frac{(5+v)}{(1+v)}+\frac{q R^{2}}{4 G_{m} h} .
$$

The first term in (20) represents the contribution to the deflection of the sheathings deformations, and the second is the contribution of the shear deformations of the filler.

In the presence of creep, equation (14) can be solved numerically by the finite difference method. To determine the creep deformations, the Euler method can be used. A detailed stepwise calculation procedure is described in [5-10].

\section{Results and Discussion}

A test problem was solved for a plate hinged along the contour at $R=1.5 \mathrm{~m}, \delta=1 \mathrm{~mm}, h=$ $8 \mathrm{~cm}, E=2 \cdot 10^{5} \mathrm{MPa}, G_{\mathrm{m}}=4.85 \mathrm{MPa}, q=2 \mathrm{kPa}$. The linear Maxwell-Thompson equation was used as the creep law, which in the case of pure shear has the form:

$$
n G \frac{\partial \gamma}{\partial t}+H \gamma=n \frac{\partial \tau}{\partial t}+\tau
$$

where $G$ and $H$ are the instantaneous and long-term shear moduli, respectively, $\mathrm{n}$ is the relaxation time, $\tau$ is the shear stress, $\gamma$ is the total shear deformation.

Representing the total shear deformation as the sum of elastic deformation and creep deformation, relation (15) can be written as:

$$
\frac{\partial \gamma^{*}}{\partial t}=\frac{1}{\kappa}\left[\left(1-\frac{H}{G}\right) \tau-H \gamma^{*}\right]
$$

where $\kappa=n G$ - coefficient of viscosity of the filler.

The calculation was carried out with the constants obtained in the work [3]: $\kappa=46.6$ M Pa.day, $H=3.17 \mathrm{MPa}$. The resulting graph of the growth of the deflection in the center 
of the plate is shown in Fig. 2. The maximum deflection at the end of the creep process was $5.36 \mathrm{~mm}$. If the creep law is given by formula (21), then it is possible to determine the deflection as $\mathrm{t} \rightarrow \infty$ by substituting the long-term modulus $H$ instead of the instantaneous modulus of filler shear $G$ into formula (20). As a result of the substitution, the value 5.35 $\mathrm{mm}$ was obtained.

To confirm the reliability of the results, finite element modeling was also performed in the LIRA software package. The middle layer of the plate was modeled by volumetric prismatic finite elements, and the sheathings were modeled by flat triangular and quadrangular shell elements. Its long-term value $H$ was substituted as the shear modulus. The isofields of vertical displacements are shown in Fig. 3. The maximum value of the deflection in the calculation in the three-dimensional formulation was $5.58 \mathrm{~mm}$, which differs from the solution obtained on the basis of the technical theory of three-layer structures by $4.1 \%$.

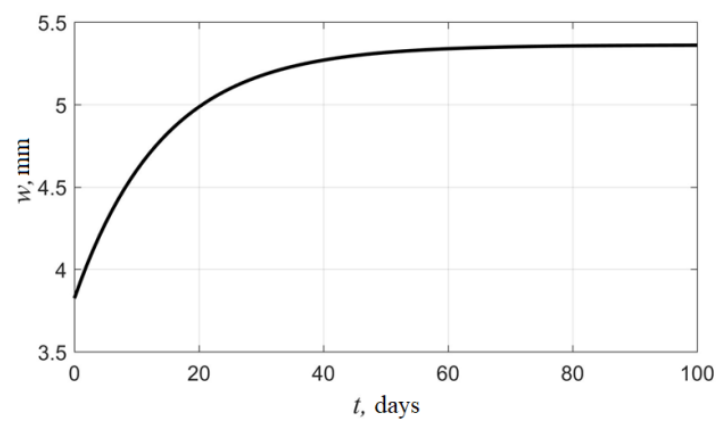

Fig. 2. Graph of deflection growth in the center of the three-layer plate

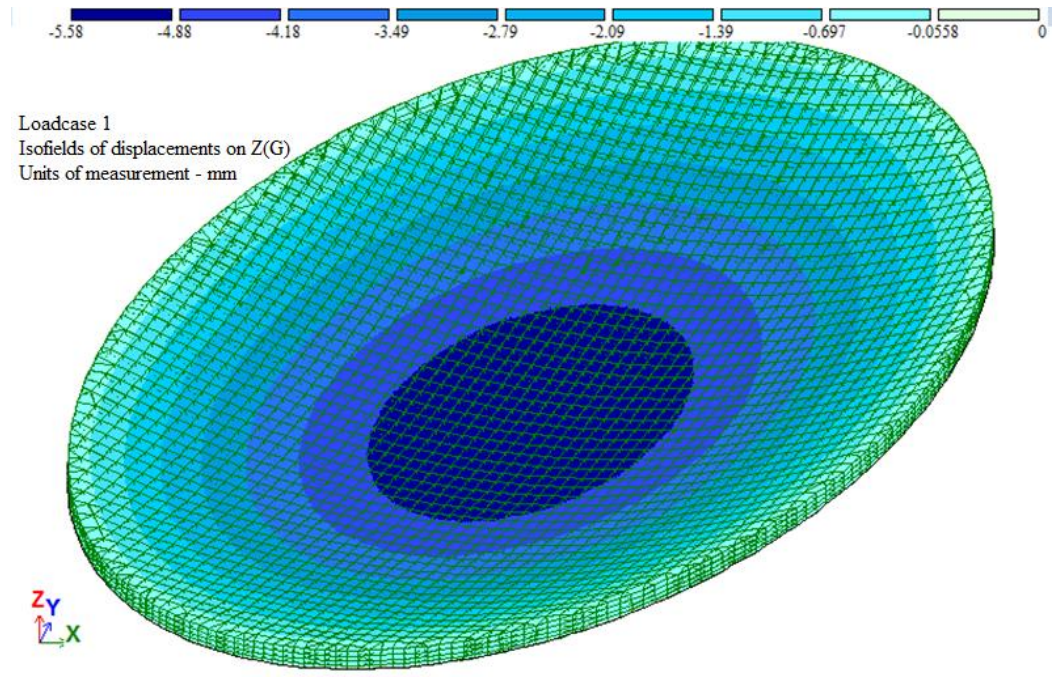

Fig. 3. I sofields of vertical displacements obtained in the LIRA software package

\section{Conclusions}

The resolving equation is obtained for calculating circular axisymmetrically loaded threelayer plates taking into account the creep of the middle layer. The problem is reduced to a 
third-order differential equation with respect to deflection. An analytical solution is obtained for the elastic problem. The calculation taking into account creep was carried out numerically by the finite difference method in combination with the Euler method. The results are compared with the LIRA software package. The discrepancy between the results is insignificant

\section{References}

1. M. Garrido et al., J ournal of Composite M aterials 48(18), 2237-2249 (2014).

2. M. Garrido, J. Correia, F. B ranco, Creep behaviour of GFRP sandwich panels with PU foam cores for civil engineering structural applications, in: 6th International Conference on FRP Composites in Civil Engineering, Roma, pp. 15-20 (2012).

3. B.M. Y azyev, A.S. Chepurnenko, A.A. Savchenko, Materials Science Forum 935, 144-149 (2018).

4. V. Chepurnenko, B. Yazyev, X. Song, MATEC Web of Conferences 129, 05009 (2017).

5. S. B. Y azyev et al., M A TEC W eb of Conferences 129, 05010 (2017).

6. V. Chepurnenko, B. Y azyev, I. Dubovitskaya, E3S W eb of Conferences 164, 02003 (2020).

7. S.V. Litvinov et al., International Polymer Science and Technology 42, 23-25 (2015).

8. S. B . Y azyev et al., IOP Conference Series: M aterials Science and E ngineering 913 (2), 022021 (2020).

9. V.S.Chepurnenko, B.M. Y azyev, International Journal for Computational Civil and Structural Engineering 15, 25-33 (2019).

10. Trush L. et al., Energy $M$ anagement of $M$ unicipal Transportation Facilities and Transport. Springer, Cham, pp. 885-893 (2017). 